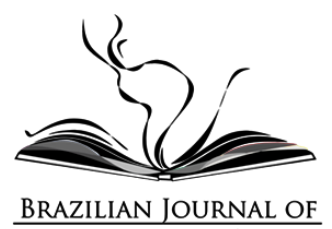

\title{
SISTEMA DE PROTECCIÓN SOCIAL COLOMBIANO: UN ANÁLISIS DE LA INTEGRACIÓN ENTRE POLÍTICAS PÚBLICAS INTERSECTORIALES'
}

\author{
SISTEMA COLOMBIANO DE PROTEÇÃO SOCIAL: UMA ANÁLISE DA \\ INTEGRAÇÃO ENTRE POLÍTICAS PÚBLICAS INTERSETORIAIS \\ COLOMBIAN SOCIAL PROTECTION SYSTEM: AN ANALYSIS OF THE \\ INTEGRATION BETWEEN INTERSECTORAL PUBLIC POLICIES
}

Cristian Camilo Rodríguez Olaya ${ }^{2}$ (iD)

Universidad Nacional de Colombia, Colombia

\begin{abstract}
Resumen: El presente artículo tiene como propósito establecer la intensidad de la intersectorialidad entre tres políticas públicas (Familias en Acción, Red Unidos y De Cero a Siempre) que se desarrollan en el marco del sistema de protección social colombiano. En el trabajo se analizan las transformaciones institucionales de las políticas señaladas y del sistema de protección social de Colombia en el periodo 2000-2020, con particular énfasis en la segunda década (2010-2020). Metodológicamente se hace uso de técnicas de recolección de información y análisis cualitativos como entrevistas, revisión documental y categorización. El artículo determina que la intensidad de la intersectorialidad entre Familias en Acción, De Cero a Siempre y Red Unidos es media-baja, lo que permite, a su vez, concluir que las políticas públicas intersectoriales constituyen un proceso que es tanto técnico como político.
\end{abstract}

Palabras clave: Políticas Públicas; Protección Social; Intersectorialidad; Programas de Transferencias Monetarias Condicionadas; Sistemas de Protección social.

Resumo: $O$ objetivo deste artigo é estabelecer a intensidade da intersetorialidade entre três políticas públicas (Familias en Acción, Red

\footnotetext{
' Este documento es producto de una investigación de maestría, realizada en El Colegio de San Luis, A.C. (México), con el objetivo de analizar la integración entre políticas intersectoriales del sistema de protección social colombiano. Este estudio fue desarrollado gracias a dos becas otorgadas por el Consejo Nacional de Ciencia y Tecnología de México (CONACYT) para estudios de maestría y movilidad internacional.

2 Politólogo, Universidad Nacional de Colombia; Filósofo, Universidad Javeriana; y Maestro en Asuntos Políticos y Políticas Públicas, El Colegio de San Luis. E-mail: c.rodriquezolaya@hotmail.com
}

Cadernos Prolam/USP-Brazilian Journal of Latin American Studies, v. 20, n. 40, p. 117-138, out. 2021

Edição especial: Trabalho e proteção social na América Latina: desenvolvimentos recentes e os desafios diante da crise pandêmica.

ISSN: 1676-6288 
Unidos e De Cero a Siempre) que se desenvolvem no âmbito do sistema de proteção social colombiano. O trabalho analisa as transformações institucionais das políticas mencionadas e do sistema de proteção social da Colômbia no período 2000-2020, com particular ênfase na segunda década (2010-2020). Metodologicamente, são utilizadas técnicas de coleta e análise de informações qualitativas, como entrevistas, revisão e análise documental e categorização. $O$ artigo determina que a intensidade da intersetorialidade entre Familias en Acción, De Cero a Siempre e Red Unidos é média-baixa, o que permite, por sua vez, concluir que as políticas públicas intersetoriais constituem um processo que é, ao mesmo tempo, técnico e político.

Palavras-chave: Políticas Públicas; Proteção Social; Intersetorialidade; Programas de Transferência Condicionada de Renda; Sistemas de Proteção Social.

Abstract: The purpose of this article is to establish the intensity of intersectoriality between three public policies (Familias en Acción, Red Unidos and De Cero a Siempre) developed that are developed within the Colombian within the framework of the Colombian social protection system framework. This study work analyzes the institutional transformations of the aforementioned policies and the social protection system of Colombia in the 2000-2020 period 2000-2020, with particular emphasis on the second decade (2010-2020). Methodologically, information gathering and qualitative analysis techniques such as interviews, documentary review and categorization are used. The article determines that the intensity of the intersectorialityintersectorality between Familias en Acción, De Cero a Siempre and Red Unidos is medium-low, which allows, in turn, to conclude that intersectoral public policies constitute a process that is both technical and political.

Keywords: Public Policies; Social Protection; Intersectoriality; Conditional Cash Transfer Programs; Social Protection Systems.

DOI:10.11606/issn.1676-6288.prolam.2021.186630

Recebido em: 07/06/2021 Aprovado em: 28/10/2021 Publicado em: 29/10/2021

\section{$1 \quad$ Introducción}

Los países de América Latina se han caracterizado en las últimas dos décadas, en términos generales, por haber desarrollado diversas políticas públicas, programas sociales y estrategias que han apuntado a la 
disminución de las desigualdades y a la reducción de la pobreza (ABRAMO; CECCHINI; MORALES, 2019). En este sentido, el grueso de los países de la región ha creado y fortalecido, de diversas maneras, sus sistemas de protección social (TASSARA; IBARRA; VARGAS, 2015), lo que no significa que dichos sistemas estén plenamente consolidados (CECCHINI; MARTÍNEZ, 2011).

En la década de 1990, el panorama de América Latina se caracterizó, en términos generales, por reestructuraciones institucionales; por la revisión crítica de las problemáticas sociales no abordadas o no resueltas; así como por debates respecto a los procesos de gestión de las políticas públicas y de los impactos deseados en el bienestar de las personas (TASSARA, 2015). Por lo tanto, el contexto señalado posibilita el hecho de que la protección social se convierta en una noción fundamental en la política social de la región, de ahí la apuesta de algunos países por formar, ampliar o reforzar sus sistemas de protección social.

Algunos de estos sistemas se han desarrollado gracias, entre otras cosas, a la construcción y ejecución de políticas públicas intersectoriales, las cuales pretenden integrar distintos sectores gubernamentales para hacer frente a problemas complejos de manera integral. Así las cosas, este artículo tiene como objetivo establecer la intensidad de la intersectorialidad entre tres políticas del sistema de protección social colombiano, con la finalidad de comprender los procesos de articulación e integración interinstitucional dentro del sistema de protección social señalado.

Por lo tanto, en este trabajo se abordan tres políticas intersectoriales de Colombia: Familias en Acción, De Cero a Siempre y Red Unidos, las cuales son parte del sistema de protección social de Colombia. De este modo, el artículo se estructura a partir del abordaje teórico de la intersectorialidad. Posteriormente, se plantea la metodología implementada en la investigación; luego se exponen los estudios de caso y finalmente los resultados y consideraciones finales. 


\section{Marco teórico: intersectorialidad}

El concepto de intersectorialidad tiene numerosos matices, dependiendo particularmente de qué premisas se adoptan o de qué se entiende por sector (CUNILL, REPETTO, BRONZO, 2015). En este orden, la noción de sector se ha asociado, por ejemplo, a las lógicas de la acción colectiva, marcando diferencias entre sectores privados, como el comercial, y el sector público (CUNILL, 2014). Por lo tanto, "la intersectorialidad está asociado a los nexos entre dichos sectores y comprende el estudio de las distintas formas de vinculación entre sectores públicos y privados" (RODRÍGUEZ-OLAYA, 2020, pág. 20).

Esta comprensión de la intersectorialidad se ha impulsado especialmente a partir de la idea de que es conveniente que las entidades privadas ofrezcan servicios públicos, con una parte de recursos públicos. Lo anterior permitiría que los riesgos y las responsabilidades son compartidos, así como las eventuales mejoras que se puedan realizar a tales servicios (HODGE; GREVE, 2017). Por otra parte, la intersectorialidad también se ha definido como la asociación entre los sectores de gobierno para una provisión de servicios públicos más eficiente, empero, fundamentalmente para aportar conjuntamente a la solución de problemas complejos. Ello significa que, debido a la multicausalidad de tales problemas, es menester realizar aproximaciones multidimensionales e integrales (DRAIBE; RIESCO, 2009).

Conforme a la concepción anterior, la intersectorialidad se refiere a la integración de diversos sectores, particularmente del ámbito gubernamental, con la finalidad de ofrecer soluciones integrales a problemas complejos (CUNILL; REPETTO; BRONZO 2015). En este sentido, la integralidad se entiende como el fundamento de las políticas intersectoriales, en la medida en que estas pretenden satisfacer demandas de las personas de naturaleza multicausal y multidimensional a partir de 
una identificación de las necesidades de la población (CECCHINI; MARTÍNEZ, 2011). Así las cosas, en este trabajo se adopta la conceptualización de intersectorialidad anteriormente expuesta.

Igualmente, la intersectorialidad en tanto concepto se compone de algunos elementos centrales que se presentan a continuación. En primer lugar, la integración, la cual se entiende aquí como el alto nivel de compromiso y colaboración entre sectores, tomando en cuenta dentro de esta la intensidad y complejidad de las relaciones (CORBETT; NOYES, 2008). En este sentido, la integración se genera en dos niveles, a saber, horizontal (entre sectores del mismo nivel administrativo, por ejemplo, el municipal) y vertical (entre sectores de niveles administrativos diferentes, por ejemplo, entre el nivel municipal y el nacional).

Como segundo elemento de la intersectorialidad puede identificarse la noción de inclusividad, la cual se refiere a la inclusión de los diversos sectores de gobierno en el denominado ciclo de las políticas públicas (CUNILL, 2005). En la inclusividad de los sectores se enmarcan cuestiones como la planificación colectiva entre sectores y/o responsables de las políticas; la formulación de propósitos compartidos; los sistemas de administración conjuntos; entre otras.

El tercer elemento constitutivo de la intersectorialidad es la mancomunidad, que se define como la unidad entre sectores respecto a las acciones, responsabilidad y recursos que se comparten para alcanzar algún objetivo colectivo o perseguido por la totalidad de los sectores y/o políticas involucradas. En este elemento se incluyen la estandarización de los procesos; sistemas de información (HORWATH; MORRISON, 2007) y presupuestos total o parcialmente compartidos; y hasta la toma de decisiones conjuntas (CUNILL; REPETTO; BRONZO, 2015).

Ahora bien, además de los tres elementos constitutivos de la intersectorialidad anteriormente expuestos, se presenta también a continuación un factor fundamental para comprender la intersectorialidad como concepto y en su operatividad, a saber, los arreglos de gobernanza 
común. Estos constituyen estructuras que "pueden ser creadas o modificadas y que emergen desde la voluntad de las entidades de gobierno o sectores, con el interés de generar dinámicas para la toma de decisiones o para llegar a consensos sobre tópicos propios de acciones intersectoriales" (RODRÍGUEZ-OLAYA, 2020, pág. 39). Por lo tanto, los arreglos intersectoriales de gobernanza común se pueden generar desde modificaciones simples (soft) en las estructuras gubernamentales existentes o hasta la formación de nuevas instancias con naturaleza intersectorial (BICHIR; CANATO, 2019).

\section{$3 \quad$ Metodología}

El presente trabajo se fundamenta en metodologías cualitativas, a través del uso de técnicas de recolección de información como entrevistas semiestructuradas y revisión documental, así como técnicas de análisis como codificación y categorización. En este sentido, el trabajo se estructura metodológicamente en tres fases de trabajo, a saber, una fase contextual, otra de recolección de información y una tercera de análisis.

Así, en la etapa contextual se identifican las características centrales del sistema de protección social colombiano, apuntando a comprender especialmente cuál es el nivel de interacción entre las políticas públicas intersectoriales estudiadas en esta investigación: Familias en Acción, Red Unidos y De Cero a Siempre. Por lo tanto, esta fase se construyó mediante entrevistas semiestructuradas a dos investigadores expertos ${ }^{31}$ en el tema estudiado y a través de revisión documental de textos oficiales del Estado colombiano sobre el sistema de protección social, sus componentes y políticas y por textos académicos e investigaciones sobre el tópico anteriormente apuntado.

\footnotetext{
${ }^{31}$ Los autores en mención son: el Doctor Carlo Tassara, profesor-investigador de la Universidad de Sapienza de Roma, quien fue entrevistado presencialmente en la ciudad de Bogotá en el año 2020, y el Oficial Superior de Asuntos Sociales que coordina la División de Desarrollo Social de la Comisión Económica para América Latina y el Caribe (CEPAL), Simone Cecchini, quien fue entrevistado de manera virtual en el año 2020.
} 
En la segunda etapa, orientada por las categorías de análisis previamente establecidas y presentadas, se recurrió a la utilización de dos técnicas de recolección de información: revisión documental y entrevistas semiestructuradas. Así, se realizaron entrevistas a altos ejecutivos del gobierno nacional de Colombia ${ }^{42}$, así como a burócratas de nivel profesional ${ }^{53}$; todos con vínculos laborales y con responsabilidades directas en el diseño e implementación de las políticas públicas analizadas en este trabajo, en el marco de la consolidación del sistema de protección social colombiano. La totalidad de las entrevistas fueron realizadas a través de plataformas virtuales (Teams, Zoom) en el año 2020.

En la tercera fase metodológica se utilizaron las siguientes técnicas de análisis: codificación y categorización. De este modo, la codificación consiste en la primera etapa del análisis en tanto pretende identificar y segmentar a un nivel primario las transcripciones de las entrevistas y los textos abordados en la recolección de información. En este orden, la categorización representa la clasificación conceptual de la información recabada en unidades de análisis establecidas, por lo que permite determinar con mayor solidez la coherencia o no de las unidades de análisis con el enfoque teórico adoptado en la investigación (GIBBS, 2012). De esta manera, las técnicas de análisis señaladas apuntaron a determinar el nivel de integración entre las políticas intersectoriales estudiadas en el escenario del sistema de protección social de Colombia. Asimismo, dichas técnicas fueron utilizadas con apoyo del software de análisis cualitativo Atlas.ti.

Con todo, en esta etapa se establecieron las dimensiones de análisis de las categorías analíticas y de sus variables, conforme a los conceptos y nociones planteados en el apartado teórico. Tal construcción metodológica

\footnotetext{
42 Los ejecutivos de alto gobierno entrevistados en esta fase fueron: Julián Torres Jiménez, director de Transferencias Monetarias Condicionadas del Departamento de Prosperidad Social (DPS); y Carolina Náder Dangond, directora de Acompañamiento Familiar y Comunitario del DPS.

53 Los burócratas entrevistados son: Jonatan Andrés Vargas Martínez, coordinador del Grupo Interno de Trabajo de Diseño metodológico y formación de la Dirección de Acompañamiento Familiar y Comunitario del DPS; Andrés Mauricio Bocanegra, coordinador de estudios técnicos del programa Familias en Acción; Tatiana Briceño Veloza, asesora de Niñez y Familia de la Subdirección General de Programas y Proyectos del DPS; y Óscar Leonardo Salcedo Colorado, coordinados del Grupo Interno de Trabajo de Implementación de la Dirección de Acompañamiento Familiar y Comunitario del DPS.
} 
se establece conforme a la literatura abordada sobre intersectorialidad, de ahí que sea particularmente importante tener presente, para una mejor comprensión de la tabla 2, el siguiente postulado: un alto grado de integración entre sectores y políticas públicas significaría entonces, una alta intensidad de la intersectorialidad.

En este sentido, se asignó a cada una de las categorías de análisis dos letras y a cada una de sus variables, una letra. Por su parte, en las dimensiones de análisis se establecieron tres niveles (alto, medio y bajo) para cada una de las variables, a los cuales se les dio un número (3, 2 y 1, conforme al orden de los niveles). De este modo, el nivel que se determina para cada variable significa el nivel de integración entre las políticas públicas del sistema de protección social colombiano, por lo que, cabe precisar, que las variables únicamente representan conjuntamente la integración entre las tres políticas. Ningún resultado corresponderá al análisis de una sola política pública.

De igual forma, es importante apuntar que la asignación de valores numéricos a los niveles de integración se realiza con el propósito de poder determinar la intensidad de la intersectorialidad entre las políticas y conforme a cada variable y categoría de análisis. Sin embargo, el primer proceso de análisis y la determinación de en qué nivel se ubica cada variable según la integración entre políticas, es resultado del análisis que dio como resultado el uso de la codificación y categorización. 
Tabla 2: Categorías analíticas, variables y dimensiones de análisis

\begin{tabular}{|c|c|c|c|c|}
\hline \multirow{3}{*}{$\begin{array}{l}\text { CATEGORÍAS } \\
\text { ANALÍTICAS }\end{array}$} & \multirow{2}{*}{$\begin{array}{l}\text { VARIABLES } \\
\text { Integración } \\
\end{array}$} & \multicolumn{3}{|c|}{ DIMENSIONES DE ANÁLISIS } \\
\hline & & \multicolumn{3}{|c|}{ Media } \\
\hline & $\begin{array}{l}\text { Valor numérico } \\
\text { asignado }\end{array}$ & 1 & 2 & 3 \\
\hline \multirow{4}{*}{$\begin{array}{c}\text { (IN) } \\
\text { Inclusividad } \\
\text { (en el ciclo de } \\
\text { políticas públicas) }\end{array}$} & $\begin{array}{c}\text { Sistemas de } \\
\text { administración } \\
\text { (S) }\end{array}$ & $\begin{array}{c}\text { Sistema } \\
\text { desarticulado }\end{array}$ & $\begin{array}{c}\text { Sistema } \\
\text { parcialmente } \\
\text { unificado }\end{array}$ & $\begin{array}{l}\text { Sistema } \\
\text { conjunto }\end{array}$ \\
\hline & Objetivos (O) & Independientes & $\begin{array}{l}\text { Parcialmente } \\
\text { compartidos y } \\
\text { difícilmente } \\
\text { identificados }\end{array}$ & $\begin{array}{l}\text { Compartidos y } \\
\text { fácilmente } \\
\text { identificados }\end{array}$ \\
\hline & Planificación $(P)$ & Independiente & Parcial & Colectiva \\
\hline & $\begin{array}{l}\text { Sistemas de } \\
\text { evaluación (E) }\end{array}$ & Independientes & $\begin{array}{l}\text { Parcialmente } \\
\text { vinculados }\end{array}$ & Unificados \\
\hline \multirow{4}{*}{$\begin{array}{c}\text { (MA) } \\
\text { Mancomunidad } \\
\text { (en las decisiones, la } \\
\text { información y los } \\
\text { recursos) }\end{array}$} & $\begin{array}{c}\text { Fondos } \\
\text { presupuestales } \\
(\mathrm{F})\end{array}$ & Independientes & $\begin{array}{l}\text { Parcialmente } \\
\text { compartidos }\end{array}$ & Compartidos \\
\hline & $\begin{array}{c}\text { Sistemas de } \\
\text { información (I) }\end{array}$ & Independientes & $\begin{array}{l}\text { Parcialmente } \\
\text { vinculados }\end{array}$ & Conjuntos \\
\hline & $\begin{array}{c}\text { Toma de } \\
\text { decisiones (D) }\end{array}$ & $\begin{array}{c}\text { Independiente, } \\
\text { con propósitos } \\
\text { sectoriales }\end{array}$ & $\begin{array}{c}\text { Fragmentada } \\
\text { con propósitos } \\
\text { mixtos }\end{array}$ & $\begin{array}{c}\text { Conjuntas con } \\
\text { un mismo } \\
\text { propósito }\end{array}$ \\
\hline & $\begin{array}{l}\text { Regularización } \\
\text { de procesos (R) }\end{array}$ & $\begin{array}{c}\text { Inexistente } \\
\text { entre sectores }\end{array}$ & Parcial & $\begin{array}{c}\text { Que permitan } \\
\text { identificar } \\
\text { unidad }\end{array}$ \\
\hline \multirow[t]{2}{*}{$\begin{array}{c}\text { (Al) } \\
\text { Arreglos } \\
\text { intersectoriales de } \\
\text { gobernanza común }\end{array}$} & $\begin{array}{l}\text { Alteraciones en } \\
\text { las estructuras } \\
\text { gubernamentales } \\
\text { (A) }\end{array}$ & Inexistentes & Parciales & Significativas \\
\hline & $\begin{array}{c}\text { Estancias } \\
\text { institucionales } \\
\text { de gobernanza } \\
\text { común }(G)\end{array}$ & Inexistentes & $\begin{array}{l}\text { Creación de } \\
\text { iniciativa } \\
\text { sectorial }\end{array}$ & $\begin{array}{l}\text { Creación } \\
\text { conjunta }\end{array}$ \\
\hline Intersectorialidad & Intensidad & Baja & Media & Alta \\
\hline
\end{tabular}

Fuente: modificado de (Rodríguez-Olaya, 2020, págs. 73-74).

Con todo, posterior a establecer el nivel de integración de cada una de las variables, se obtiene el promedio ponderado entre las variables de cada una de las categorías, el cual representa la intensidad de la intersectorialidad entre las políticas públicas analizadas del sistema de protección social. De esta manera, a continuación, se presentan las fórmulas determinadas para alcanzar el objetivo de la investigación, las cuales representan las variables en letras, conforme a la tabla 2.

$$
\mathrm{IN}=\frac{S+O+P+E}{4} \quad \mathrm{MA}=\frac{F+I+D+R}{4} \quad \mathrm{Al}=\frac{A+G}{2}
$$

De este modo, una vez se obtenga el promedio ponderado para cada una de las categorías analíticas, se realiza, con la finalidad de determinar la intensidad de la intersectorialidad entre las tres políticas públicas 
abordadas, una operación aritmética similar a la presentada en fórmulas anteriores, empero, con el resultado hallado en cada una de las categorías, como se muestra a continuación:

$$
\text { II (Intensidad de la intersectorialidad })=\frac{I N+M A+A I}{3}
$$

Así las cosas, los resultados obtenidos en la última fórmula presentada (II) se ubican en la gráfica uno, dividida en los siguientes niveles de intensidad de la intersectorialidad: bajo, medio-bajo, medio-alto y alto. Ello se plantea así, con el propósito de poder definir en mayor medida los matices que pudieran reflejarse de la intersectorialidad entre las políticas Familias en Acción, Red Unidos y De Cero a Siempre.

Por lo tanto, se establecen cuatro niveles en que se valora la intensidad de la intersectorialidad. Empero, si bien de 0-1 la intensidad de la intersectorialidad entre las políticas sería nula, se parte del hecho de que las tres políticas seleccionadas en este trabajo tienen fundamento $u$ orientaciones hacia la intersectorialidad (de ahí porqué se eligieron para el estudio), por lo menos hay algún grado mínimo de intensidad de la intersectorialidad entre dichas políticas. Por su parte, el nivel bajo se estableció numéricamente entre 1-1.49; el nivel medio-bajo entre 1.50-1.99; el medio-alto entre 2-2.49; y el alto entre 2.50-3.

\section{Sistema de protección social colombiano}

La protección social ha sido comprendida en términos de las instituciones públicas, programas y normas que tienen como propósito la protección de las personas y de sus hogares de la pobreza y privaciones socioeconómicas básicas (BARRIENTOS; SHEPHERD, 2003). Autores como Simone Cecchini y Rodrigo Martínez (2011) definen la protección social como una garantía de la ciudadanía con enfoque universal, cuestión que no significa desconocer la necesidad de adoptar instrumentos focalizados y diferenciados. De este modo, la protección social incluye acciones contra 
el desempleo o las injusticias laborales; crisis y/o contingencias económicas y sociales; dificultades en los ciclos de vida como las que pueden encontrarse en la maternidad o en la vejez; o problemas sanitarios públicos, entre otros.

Desde mediados de la década de 1990 y hasta el presente, en Colombia se ha avanzado en la formación y consolidación de un sistema de protección social, aún con muchos desafíos. Para ello, se han articulado diversas intervenciones de carácter público que pretenden disminuir y combatir los distintos riesgos a los que están expuestas las poblaciones (ACOSTA; FORERO; PARDO, 2015). De igual manera, si bien el SPS colombiano ha estado en permanente consolidación, fue definido por el Congreso de la República de Colombia en 2002 "como el conjunto de políticas públicas orientadas a disminuir la vulnerabilidad y a mejorar la calidad de vida de los colombianos, especialmente de los más desprotegidos. Para obtener como mínimo el derecho a: la salud, la pensión y el trabajo" (COLOMBIA, 2002, art. 1, pág. 1).

Así las cosas, el sistema de protección social colombiano actual se estructura, como parte de un proceso complejo de ajustes institucionales, en cinco ejes fundamentales que le posibilitan dar prioridad a sus intervenciones, a saber, la seguridad social integral; el acceso a activos físicos y financieros; la formación en capital humano; el sistema social de riesgo; y el sistema de promoción social. De esta forma, la seguridad social con vocación universal fomenta el aseguramiento de la población a la salud, pensión y riesgos laborales, bien sea de manera subsidiada o como cotizantes (TASSARA; IBARRA; VARGAS, 2015).

Por su parte, el eje de promoción social está orientado a la población más vulnerable y pobre del país, la cual demanda múltiples acciones para mejorar sustancialmente su condición. Este eje demanda un enfoque integral que contemple las diversas causas de la pobreza y la vulnerabilidad $\mathrm{y}$, por lo tanto, no consiste en una aproximación asistencialista (TASSARA; 
IBARRA; VARGAS, 2015). Al respecto, un informe de la CEPAL señala que en Colombia

\begin{abstract}
Se ha avanzado del asistencialismo a la promoción social y los debates actuales giran en torno al incremento de la calidad, la cobertura de los informantes y el aseguramiento de ingresos en la vejez en un marco de derechos para toda la población. La expresión de ese objetivo es la construcción gradual de un SPS gradual (ACOSTA; FORERO; PARDO, 2015, pág. 9).
\end{abstract}

Ahora bien, en el componente de promoción social se encuentran algunos de los programas sociales más importantes del país, como Familias en Acción o Red Unidos. Por lo tanto, a continuación, se abordarán analíticamente las dos políticas públicas intersectoriales mencionadas anteriormente, además de la Política De Cero a Siempre, la cual también se desarrolló en el marco del sistema de protección social de Colombia.

\title{
4.1 Familias en Acción
}

Familias en Acción es un programa de transferencias monetarias condicionadas (PTMC) que entrega un monto económico de manera periódica a un número significativo de familias en situación de pobreza. Este incentivo se da, con el propósito y la condición, de que los niños, niñas y adolescentes asistan a la escuela o colegio regularmente, así como que los niños efectivamente sean llevados a controles de salud cada determinado tiempo (COLOMBIA, 2019).

De igual manera, este PTMC -el más grande de Colombia- se ha desarrollado en tres etapas: de 2000-2006, en la que Familias en Acción operó en municipios rurales y de menos de 100.000 habitantes en el país. Esto con apoyo financiero del Banco Interamericano de Desarrollo-BID y del Banco Mundial (MINA, 2013). En la segunda etapa, de 2007-2011, se amplió la cobertura del programa de Familias en Acción a municipios de más de 100.000 personas. Además, se establecieron estrategias para vincular a familias indígenas al programa, así como con familias beneficiarias del, entonces naciente, programa Red Unidos (DPS, 2019). 
La tercera fase de Familias en Acción (2012-2021), se destaca por la creación del Departamento de Prosperidad Social (DPS), entidad que pasó a liderar la operación del PTMC mencionado y que, implicó que el DPS se posesionara en la institución de nivel nacional con mayores responsabilidades en el combate a la pobreza, así como una de las centrales en el sistema de protección social del país. De igual manera, la ley 1532 de 2012 otorgó a Familias en Acción fundamento jurídico para su cobertura total en el territorio nacional y para ser un programa de Estado permanente (COLOMBIA, 2012).

\subsection{Red Unidos}

Es una estrategia nacional que vincula a diversas entidades del Estado colombiano, con la finalidad de disminuir los niveles de pobreza extrema en el país (DNP, 2021). Por lo tanto, Red Unidos, más que un programa social, constituye una amplia oferta de diversos programas sociales o de beneficios del Estado que apuntan a incrementar las condiciones de los hogares más pobres de Colombia.

Red Unidos tiene su origen en la propuesta que formuló en 2004 la Misión para el Diseño de una Estrategia para la Reducción de la Pobreza y la Desigualdad (MERPD), la cual indicó que era menester construir una red de protección social que tuviera como objetivo central la superación de la pobreza. En este sentido, se crea la primera versión de Red Unidos, denominada entonces como Red Juntos (CONPES, 2006), la cual pasa años después a ser coordinada por la Agencia Nacional para la Superación de la Pobreza Extrema (ANSPE), así como cambia su nombre a Red Unidos.

Así las cosas, la Red Unidos se concreta en la Estrategia Unidos que es "el conjunto de acciones a mejorar las condiciones de vida de los hogares en situación de extrema pobreza, en función de dimensiones y logros que delimitan su intervención" (DPS, 2015, pág. 10). Por lo tanto, 
dicha estrategia opera mediante el acompañamiento familiar a hogares en condición de pobreza extrema, permitiendo un acceso preferente y oportuno, conforme a sus necesidades, de la oferta social pública y privada de la que puedan beneficiarse las personas (DPS, 2020).

Por otro lado, en el año 2016 la Ley 1785 le otorga estabilidad presupuestaria e institucional a la Red Unidos. Igualmente, el Plan Nacional de Desarrollo (PND) 2018-2022 reconoce que el potencial de la Red Unidos es ser:

La puerta de ingreso de la oferta estatal para la superación de la pobreza extrema, de tal forma que el acompañamiento familiar y comunitario se convierta en una plataforma para hacer eficiente la implementación de los programas sociales para la superación de la pobreza extrema (DNP, 2019, pág. 160).

\subsection{De Cero a Siempre}

Esta es la estrategia nacional de atención integral a la primera infancia y constituye, por lo tanto, la política de Estado para este grupo poblacional. De esta forma, De Cero a Siempre debe desarrollar acciones que apunten al fortalecimiento de las capacidades de protección, afecto y cuidado de la primera infancia (DNP, 2011, pág. 257).

Por lo tanto, De Cero a Siempre nace a inicios de la década 2010-2020, conforme a la necesidad de ofrecer y garantizar una atención de tipo integral a la primera infancia (esto es, de cero hasta los casi seis años), que se caracterice por la coordinación intersectorial, así como por la integración de diversos programas sociales sobre dicho grupo poblacional (TORRADO et al, 2017).

En este sentido, como resultado del impulso de la Política de Cero a Siempre en el Plan Nacional de Desarrollo de Colombia 2010-2014 (DNP, 2011, pág. 258), se crea con el Decreto 4875 la Comisión Intersectorial para la Atención Integral de la Primera Infancia (CIPI) (MINISTERIO DE JUSTICIA, 2011). Igualmente, el Plan Nacional de Desarrollo 2014-2018 mantiene y 
fortalece la capacidad intersectorial operativa de la política De Cero a Siempre (DNP, 2015, págs. 66-67).

Con todo, De Cero a Siempre se convierte en la Política de Estado en favor de la primera infancia con la Ley 1804 de 2016, cuestión que le otorga estabilidad institucional y presupuestal. Lo anterior, no obstante, ha sido cuestionado por autores como (TORRADO, 2017) al señalar que existe todavía, en el caso De Cero a Siempre, una significativa desarticulación entre los diversos actores del Estado en la atención a la primera infancia, lo que permitiría argumentar que aún el sistema de protección social colombiano no es lo suficientemente integral como se plantea institucionalmente.

\section{$5 \quad$ Resultados}

Como resultado del análisis cualitativo de la integración entre Familias en Acción, Red Unidos y De Cero a Siempre, se obtuvo para la categoría analítica "inclusividad" una intensidad de la intersectorialidad de nivel medio, como se puede observar en la tabla 3 y en el promedio ponderado alcanzado con esta categoría.

Tabla 3 Resultados inclusividad entre Familias en Acción, De Cero a Siempre y Red Unidos

\begin{tabular}{|c|c|c|c|c|}
\hline \multirow{2}{*}{$\begin{array}{c}\text { Categorías } \\
\text { analíticas }\end{array}$} & Variables & Resultado & $\begin{array}{c}\text { Nivel de } \\
\text { integración }\end{array}$ & $\begin{array}{c}\text { Valor numérico } \\
\text { correspondiente }\end{array}$ \\
\cline { 2 - 5 } & $\begin{array}{c}\text { Sistemas de } \\
\text { administración } \\
(\mathrm{S})\end{array}$ & $\begin{array}{c}\text { Sistema } \\
\text { parcialmente } \\
\text { unificado }\end{array}$ & Media & 2 \\
\cline { 2 - 5 } $\begin{array}{c}\text { Inclusividad } \\
\text { (en el ciclo de } \\
\text { las políticas } \\
\text { públicas) }\end{array}$ & Objetivos (O) & $\begin{array}{c}\text { Compartidos y } \\
\text { fácilmente } \\
\text { identificados }\end{array}$ & Alta & 3 \\
\cline { 2 - 5 } & Planificación (P) & Parcial & Media & 2 \\
\cline { 2 - 5 } & $\begin{array}{c}\text { Sistemas de } \\
\text { evaluación (E) }\end{array}$ & Independientes & Baja & 1 \\
\hline
\end{tabular}

$$
\mathrm{IN}=\frac{S+O+P+E}{4} \quad=\quad I N=\frac{2+3+2+1}{4}=2,0
$$


En el caso de la categoría "mancomunidad" se logró establecer que, conforme a las cuatro variables que la integran, la intensidad de la intersectorialidad es media (ver tabla 4 y fórmulas de MA).

Tabla 4 Resultados mancomunidad entre Familias en Acción, De Cero a Siempre y Red Unidos

\begin{tabular}{|c|c|c|c|c|}
\hline \multirow[b]{2}{*}{$\begin{array}{c}\text { Categorías } \\
\text { analíticas }\end{array}$} & \multicolumn{4}{|c|}{ Dimensiones de análisis } \\
\hline & Variables & Resultado & $\begin{array}{c}\text { Nivel de } \\
\text { integración }\end{array}$ & $\begin{array}{l}\text { Valor numérico } \\
\text { correspondiente }\end{array}$ \\
\hline \multirow{4}{*}{$\begin{array}{c}\text { (MA) } \\
\text { Mancomunidad } \\
\text { (en las } \\
\text { decisiones, la } \\
\text { información y los } \\
\text { recursos) }\end{array}$} & $\begin{array}{c}\text { Fondos } \\
\text { presupuestales } \\
(\mathrm{P})\end{array}$ & $\begin{array}{l}\text { Parcialmente } \\
\text { compartidos }\end{array}$ & Media & 2 \\
\hline & $\begin{array}{c}\text { Sistemas de } \\
\text { información (S) }\end{array}$ & $\begin{array}{c}\text { Parcialmente } \\
\text { vinculados }\end{array}$ & Media & 2 \\
\hline & $\begin{array}{c}\text { Toma de } \\
\text { decisiones (D) }\end{array}$ & $\begin{array}{c}\text { Fragmentada } \\
\text { con propósitos } \\
\text { mixtos }\end{array}$ & Media & 2 \\
\hline & $\begin{array}{l}\text { Regularización } \\
\text { de procesos (R) }\end{array}$ & Parcial & Baja & 2 \\
\hline
\end{tabular}

$$
\mathrm{MA}=\frac{P+S+D+R}{4} \quad=\quad M A=\frac{2+2+2+2}{4}=2,0
$$

Por su parte, en la categoría de "arreglos de gobernanza común" se obtuvo, mediante el análisis cualitativo de las variables, una intensidad de la intersectorialidad de 1.5, siguiendo los resultados de la tabla 5. Este resultado representa una intensidad de nivel bajo-medio.

Tabla 5 Resultados arreglos de gobernanza común entre Familias en Acción, De Cero a Siempre y Red Unidos

\begin{tabular}{|c|c|c|c|c|}
\hline \multirow[b]{2}{*}{$\begin{array}{c}\text { Categorías } \\
\text { analíticas }\end{array}$} & \multicolumn{4}{|c|}{ Dimensiones de análisis } \\
\hline & Variables & Resultado & $\begin{array}{c}\text { Nivel de } \\
\text { integración }\end{array}$ & $\begin{array}{l}\text { Valor numérico } \\
\text { correspondiente }\end{array}$ \\
\hline \multirow{2}{*}{$\begin{array}{l}(\mathrm{Al}) \\
\text { Arreglos de } \\
\text { gobernanza } \\
\text { común }\end{array}$} & $\begin{array}{l}\text { Alteraciones en } \\
\text { las estructuras } \\
\text { gubernamentales } \\
\text { (A) }\end{array}$ & Inexistentes & Baja & 1 \\
\hline & $\begin{array}{c}\text { Estancias } \\
\text { institucionales } \\
\text { de gobernanza } \\
\text { común (G) }\end{array}$ & $\begin{array}{c}\text { Creación de } \\
\text { iniciativa } \\
\text { sectorial }\end{array}$ & Media & 2 \\
\hline
\end{tabular}
$A I=\frac{A+G}{2}$
$=$
$A I=\frac{1+2}{2}=1,5$ 
Por lo tanto, como resultado conjunto del promedio ponderado del resultado cuantitativo de la intensidad de la intersectorialidad entre las tres categorías analíticas, se determinó que Familias en Acción, Red Unidos y De Cero a Siempre, tienen una intensidad de la intersectorialidad de nivel bajo-medio, o de 1,83, conforme a la tabla 6.

Tabla 6 Resultados integración de la integración por categorías analíticas

\begin{tabular}{|c|c|}
\hline Categorías analíticas & Resultado cuantitativo \\
\hline Inclusividad & 2,0 \\
\hline Mancomunidad & 2,0 \\
\hline $\begin{array}{c}\text { Arreglos de gobernanza } \\
\text { común }\end{array}$ & 1,5 \\
\hline Resultado total & 1,83 \\
\hline
\end{tabular}

\section{Consideraciones finales}

Como se mostró en el anterior acápite, el análisis de la integración entre las tres políticas públicas estudiadas del sistema de protección social de Colombia arrojó como resultado una intensidad de la intersectorialidad de nivel medio-bajo. No obstante, más allá de los resultados mencionados en el punto anterior, es posible llegar a algunas conclusiones teórico-conceptuales, metodológicas y empíricas a partir de la investigación realizada, tal como se exponen a continuación.

En primer lugar, es válido concluir que las políticas públicas intersectoriales efectivamente se pueden comprender como un proceso político y técnico. Lo anterior conforme al entendimiento de que constituyen un proceso en tanto son políticas públicas que buscan la integración con otras políticas y/o sectores para combatir problemas complejos, lo que significa que no se definen como intersectoriales únicamente por ser así diseñadas, sino porque se construyen de tal manera justamente en el ejercicio cíclico de las políticas públicas.

Asimismo, dichas políticas se pueden comprender como proceso político, puesto que, para su efectiva integración demandan de consensos 
y voluntades políticas reales que posibiliten una cabal implementación, incluso más que en otro tipo de políticas, al requerir estas por su diseño mayores niveles de articulación intersectoriales. De igual modo, son también un proceso técnico en tanto operan a partir de diversos mecanismos de integración e instrumentos de política pública que demandan un significativo nivel de tecnicismo.

En segundo lugar, es posible argumentar que las políticas públicas intersectoriales efectivamente pretenden, en el marco del sistema de protección social colombiano, enfrentar problemas complejos de forma integral. Esto se puede sostener, dado que, por lo menos en su diseño y en varios de los instrumentos de política pública analizados en este trabajo, se logra identificar que tales políticas parten de una multicausalidad de los problemas y buscan ofrecer contra estos abordajes y soluciones multidimensionales.

En tercer lugar, conforme a lo sustentado en el estudio es válido apuntar que las políticas públicas intersectoriales, en su diseño, deben pretenden formular y establecer los mecanismos técnicos que permitan tener mayores niveles de integración y, con ello, niveles más altos de intensidad de la intersectorialidad. Sin embargo, es imperativo que para que dichos mecanismos de integración realmente funcionen, siguiendo lo señalado en la primera conclusión, se tenga también consenso y voluntad política, además de la robustez técnica.

Con todo, entender a las políticas intersectoriales en tanto procesos político-técnicos permite comprender que estas se dan en escenarios políticos que es preciso identificar y analizar, en términos de actores, desafíos e intereses. De ahí que, por lo tanto, el desarrollo de los sistemas de protección social se deba entender desde una perspectiva técnica -respecto a los mecanismos e instrumentos de integración entre sectores y de oferta de servicios- $y$, sobre todo, desde un enfoque político.

\section{Referencias}


ABRAMO, Laís; CECCHINI, Simone; MORALES, Beatriz. Programas sociales, superación de la pobreza e inclusión laboral. Aprendizajes desde América Latina y el Caribe. Santiago de Chile, Chile: CEPAL, 2019. Disponible en: https://repositorio.cepal.org/bitstream/handle/11362/44602/1/S1900005_es.p df. Accedido en: 23 oct. 2021

ACOSTA, Olga; FORERO, Nohora; PARDO, Renata. Sistema de protección social de Colombia. Avances y desafíos. Santiago de Chile: CEPAL, 2015. Disponible https://repositorio.cepal.org/bitstream/handle/11362/37882/1/S1421114_es.pdf Accedido en: 23 oct. 2021

BARRIENTOS, Armando; SHEPHERD, Andrew. Chronic Poverty and Social Protection. In: Conference on Chronic Poverty. Annals. University of Manchester, Manchester: 2003.2 Disponible en: https://www.researchgate.net/publication/233136267_Chronic_Poverty_and_ Social_Protection_Introduction/link/00463519b8ace80dc5000000/downloa d. Accedido en: 23 oct. 2021

BICHIR, Renata; CANATO, Pamela. Solucionando problemas complexos? Desafios da implementação de políticas intersectoriais. In: Implementando desigualdades: reprodução de desigualdades na implementação de políticas públicas. Río de Janeiro: IPEA, 2019, p. 243-265. Disponible en: https://www.ipea.gov.br/portal/images/stories/PDFs/livros/livros/190527_livro _implementando_desigualdades_reproducao_de_desigualdades_Cap9.pdf Accedido en: 23 oct. 2021

CECCHINI, Simone; MARTÍNEZ, Rodrigo. Protección social inclusiva en América Latina. Una mirada integral, un enfoque de derechos. Santiago de Chile,: CEPAL, 2011, Disponible en: https://repositorio.cepal.org/bitstream/handle/11362/2593/1/S2011914_es.pdf Accedido en: 23 oct. 2021

COLOMBIA (Congreso de la República de Colombia). Ley 789 de 2002. Bogotá, 2002. Disponible en: http://www.secretariasenado.gov.co/senado/basedoc/ley_0789_2002.html

COLOMBIA (Congreso de la República de Colombia). Ley 1532 de 2012. Bogotá. $2012 . \quad$ Disponible en: http://www.secretariasenado.gov.co/senado/basedoc/ley_1532_2012.html

COLOMBIA (Congreso de la República de Colombia). Ley 1948 de 2019. Bogotá, $2019 . \quad$ Disponible en: http://www.secretariasenado.gov.co/senado/basedoc/ley_1948_2019.html Accedido en: 23 oct. 2021

CONPES (Consejo Nacional de Política Económica y Social). Documento CONPES Social 102 de 2006. Red de Protección Social contra la Extrema Pobreza. Departamento Nacional de Planeación, 2006. Disponible en: 
https://colaboracion.dnp.gov.co/CDT/Conpes/Social/102.pdf Accedido en: 23 oct. 2021

CORBETT, Thomas; NOYES, Jennifer. Human Services Systems Integration: A Conceptual Framework. In: Annual Meeting of the Association for Public Policy Analysis and Management. Annals. Washington, DC, 2008. Disponible en: https://irp.wisc.edu/publications/dps/pdfs/dp133308.pdf Accedido en: 23 oct. 2021

CUNILL, Nuria. La intersectorialidad en el gobierno y gestión de la política social. Documento presentado. In: Congreso Internacional del CLAD sobre la Reforma del Estado y de la Administración Pública, 10; 18 - 21 Oct. 2005. Santiago de Chile. Anais, Santiago, Chile, p. 1-52 Disponible en: https://repositoriocdim.esap.edu.co/bitstream/handle/123456789/8719/64421.pdf?sequence=1\&isAllowed=y . Accedido en: 23 oct. 2021

CUNILL, NURIA. La intersectorialidad en las nuevas políticas sociales. Un acercamiento analítico-conceptual. Gestión y Política Pública, 23(1), 2014, p. 5-46. Disponible en: http://www.scielo.org.mx/pdf/gpp/v23n1/v23nlal.pdf Accedido en: 23 oct. 2021

CUNILL, Nuria; REPETTO, Fabián; BRONZO, Carla. Coordinación intersectorial pro integralidad de las instituciones de protección social. In: CECCHINI, Simone et al. (Edits.), Instrumentos de protección social. Caminos latinoamericanos hacia la universalización. Santiago de Chile: CEPAL, 2015, p. 407-444. Disponible en: https://repositorio.cepal.org/bitstream/handle/11362/39678/S1500279_es.pdf? sequence=1\&isAllowed=y Accedido en: 23 oct. 2021

DNP (Departamento Nacional de Planeación-DNP). Plan Nacional de Desarrollo 2010- 2014. Prosperidad para todos. Bogotá: DNP, 2011. Disponible

en: https://colaboracion.dnp.gov.co/CDT/PND/PND2010-2014\%20Tomo\%20I\%20 CD.pdf Accedido en: 23 oct. 2021

DNP (Departamento Nacional de Planeación). Plan Nacional de Desarrollo 2014- 2018. Todos por un nuevo país. Bogotá: DNP, 2015. Disponible en: https://colaboracion.dnp.gov.co/CDT/PND/PND\%202014-2018\%20Tomo\%201 \%2Ointernet.pdf Accedido en: 23 oct. 2021

DNP (Departamento Nacional de Planeación). Bases del Plan Nacional de Desarrollo 2018-2022. Bogotá: DNP, 2019. Disponible en: https://colaboracion.dnp.gov.co/CDT/Prensa/BasesPND2018-2022n.pdf Accedido en: 23 oct. 2021

DNP (Departamento Nacional de Planeación). Red Unidos para Superación de la Pobreza Extrema. Bogotá: DNP, 2021. Disponible en: https://www.dnp.gov.co/programas/desarrollo-social/pol\%C3\%ADticas-social es-transversales/Paginas/red-unidos-para-superacion-de-la-pobreza-extre ma.aspx Accedido en: 23 oct. 2021 
DPS (Departamento de Prosperidad Social). Manual Operativo de la Estrategia Red Unidos. Bogotá: Agencia Nacional para la Superación de la Pobreza $\quad$ Extrema-ANSPE, $2015 . \quad$ Disponible en: https://www.ucn.edu.co/programas-extension/Documents/unidos/MANUAL -OPERATIVO.pdf Accedido en: 23 oct. 2021

DPS (Departamento de Prosperidad Social). Manual Operativo Familias en Acción. Bogotá: $\quad 2019 . \quad$ DPS, Disponible en: http://centrodedocumentacion.prosperidadsocial.gov.co/2020/Familias-enAccion/Manuales/M-GI-TM-3-MANUAL-OPERATIVO-FAMILIAS-EN-ACCIO\%C C\%81N-V5.pdf . Accedido en: 23 oct. 2021

DPS (Departamento de Prosperidad Social). Acompañamiento Familiar y Comunitario. Bogotá: $2020 . \quad$ Disponible en: https://dps2018.prosperidadsocial.gov.co/ent/gen/prg/Paginas/Acompa\%C3 \%Blamiento-Familiar-y-Comunitario.aspx Accedido en: 23 oct. 2021

DRAIBE, Sônia; RIESCO, Manuel. El Estado de bienestar social en América Latina. Una nueva estrategia de desarrollo. Madrid: Fundación Carolina, 2009.

https://www.fundacioncarolina.es/wp-content/uploads/2014/08/DT31.pdf Accedido en: 23 oct. 2021

GIBBS, Graham El análisis de datos cualitativos en investigación cualitativa. Madrid: Ediciones Morata, 2012. Disponible en: https://dpp2016blog.files.wordpress.com/2016/08/graham-gibbs-el-anc3allis is-de-datos-cualitativos-en-investigacic3b3n-cualitativa.pdf Accedido en: 23 oct. 2021

HODGE, Graeme; GREVE, Carsten. On Public-Private Partnership Performance: A Contemporary Review. In: Public Works Management \& Policy, 22(1), 2017, p. 55-78. DOl.org/10.1177/1087724X16657830 Accedido en: 23 oct. 2021

HORWATH, Jean; MORRISON, Tony. Collaboration, integration and change in children's services: Critical issues and key ingredients. In: Child Abuse \& Neglect, 31, 2007, p. 55-69. DOI.org/10.1016/j.chiabu.2006.01.007 Accedido en: 23 oct. 2021

MINA, Lucía. Sistemas de protección social en América Latina y el Caribe. Colombia. Santiago de Chile: CEPAL, 2013. Disponible en: https://repositorio.cepal.org/bitstream/handle/11362/4078/1/s2013331_es.pdf Accedido en: 23 oct. 2021

MINISTERIO DE JUSTICIA. Decreto 4875 de 2011. Bogotá: Sistema Único de Información Normativa, 2011. Disponible en: http://www.suin-juriscol.gov.co/viewDocument.asp?ruta=Decretos/1553065 Accedido en: 23 oct. 2021 
REPETTO, F. Protección social en América Latina: la búsqueda de una integralidad con enfoque de derechos. Revista del CLAD Reforma y Democracia, $\quad$ 47, 2010. Disponible en: https://www.redalyc.org/pdf/3575/357533679004.pdf Accedido en: 23 oct. 2021

RODRÍGUEZ-OLAYA, Cristian. Políticas públicas intersectoriales: un análisis de políticas para la superación de la pobreza en Colombia. Tesis (Maestría en políticas públicas). San Luis Potosí: El Colegio de San Luis, A.C. 2020.

Disponible en: https://colsan.repositorioinstitucional.mx/jspui/bitstream/1013/966/1/Pol\%C3 \%ADticas\%20p\%C3\%BAblicas\%20intersectoriales.pdf Accedido en: 23 oct. 2021

TASSARA, Carlo. Políticas Públicas de protección social y lucha contra la pobreza en Colombia: logros y desafíos. Papel Político, 20(2), 2015, p. 323-351. DOl.org/10.11144/ Javeriana.papo20-2.ppps.

TASSARA, Carlo; IBARRA, Antonio; VARGAS, Luis. Protección social y lucha contra la pobreza en Brasil, Colombia y Chile. ¿Graduarse de los PTC o salir de la pobreza? Madrid: Programa EUROsociAL, 2015. Accedido en: 23 oct. 2021

TORRADO, María. et al. La política pública para la primera infancia frente a la desigualdad social en Colombia. In: DURÁN, Ernesto; TORRADO, María (Edits), Políticas de infancia y adolescencia ¿Camino a la equidad? Bogotá: Universidad Nacional de Colombia, 2017, p. 87-116. Accedido en: 23 oct. 2021 